Pacific Journal of Mathematic 


\title{
A UNIQUENESS THEOREM FOR TEMPERED INVARIANT EIGENDISTRIBUTIONS
}

\author{
R. A. HERB
}

Let $G$ be a real reductive Lie group and $\pi$ a tempered invariant eigendistribution on $G$. Given a natural ordering on the set of conjugacy classes of Cartan subgroups of $G$, $\pi$ is called extremal if it has a unique maximal element in its support. T. Hirai has proved for a restricted class of real simple Lie groups that if $\pi$ is extremal and satisfies certain regularity conditions, it is uniquely determined by its restriction to the maximal element in its support. The purpose of this paper is to show that Hirai's theorem is true without restriction of the type of $G$.

1. Introduction. Let $G$ be a connected, acceptable, real reductive Lie group with compact center. Let $\pi$ be a tempered invariant eigendistribution on $G$. Then $\pi$ can be realized as a locally summable function, $\pi^{\prime}$, analytic on the dense open set $G^{\prime}$ of regular elements of $G$. [1] This function is uniquely determined by its restrictions to a complete set of representatives of $\operatorname{Car}(G)$, the set of conjugacy classes of Cartan subgroups of $G$.

The function $\pi^{\prime}$ can be quite complicated on the various Cartan subgroups. However, there is a natural ordering on $\operatorname{Car}(G)$ such that, if $[H] \in \operatorname{Car}(G)$ is a maximal element for which $\left.\pi^{\prime}\right|_{H} \neq 0$, then there exist functions $\varepsilon_{R}^{H}$ and $\Delta^{H}$ on $H$ such that $k^{H}=\left.\varepsilon_{R}^{H} \Delta^{H} \pi^{\prime}\right|_{H}$ is analytic on all of $H$ and is given by a simple Weyl character type formula [5, vol. II, p. 60-62]. Thus it is useful to know when $\pi$ is uniquely determined by the restrictions of $\pi^{\prime}$ to maximal Cartan subgroups in its support.

For example, if $\pi$ is the character of a discrete series representation of $G$, then $G$ has a compact Cartan subgroup $B$ which belongs to the unique maximal conjugacy class in $\operatorname{Car}(G)$. Harish-Chandra proved in [2] that $\pi$ is the unique tempered invariant eigendistribution with given eigenvalue and given formula on $B$.

In two recent papers $[3,4]$, Hirai studies the space of tempered invariant eigendistributions on $G$, and proves a theorem which is a natural generalization of the theorem of Harish-Chandra. However, there is a crucial lemma for this theorem which is only stated for $G$ a simple real Lie group of classical type or of type $G_{2}$. Hirai doesn't give a proof of this lemma, but claims it is proved by long but elementary case by case arguments.

The purpose of this paper is to give a general proof of Hirai's 
lemma valid for any reductive real Lie group. As a consequence, Hirai's theorem on uniqueness of tempered invariant eigendistributions can be proved without restriction of the type of $G$.

Section 2 of this paper gives the details of Hirai's results and their extensions. Most notation and definitions follow [3, 4]. Section 3 gives the proof of Lemma 5 of $\S 2$.

2. Uniqueness of tempered invariant eigendistributions. Let $G$ be as in $\S 1$, with Lie algebra $\mathfrak{Y}$. Let $A_{T}(\lambda)$ be the set of all tempered invariant eigendistributions on $G$ with eigenvalue $\lambda$. Thus, an element $\pi$ of $A_{T}(\lambda)$ is a distribution on $C_{c}^{\infty}(G)$ such that:

(i) $\pi$ is invariant under inner automorphisms of $G$;

(ii) $Z \pi=\lambda(Z) \pi$ where $Z \in \mathscr{Z}$, the center of the universal enveloping algebra of the complexification $\mathfrak{Y}_{c}$ of $\mathfrak{Y}$ and $\lambda$ is a homomorphism from $\mathscr{z}$ to $C$;

(iii) $\pi$ extends continuously to a distribution on $\mathscr{C}(G)$, the Schwartz space of $G$.

Let $\operatorname{Car}(G)$ and $\operatorname{Car}(\mathfrak{Y})$ denote the sets of all conjugacy classes of Cartan subgroups of $G$ and Cartan subalgebras of $\bigvee$ respectively. Then there is an order on $\operatorname{Car}(\mathfrak{Y )})$, which can be lifted to an order of $\operatorname{Car}(G)$, given as follows. Let $\mathfrak{A}$ and $\mathfrak{B}$ be Cartan subalgebras of $\mathfrak{Y}$. We say [ㅈ] $<[\mathfrak{B}]$ if there is a real root $\alpha$ of $\mathfrak{A}$ such that $\nu(\mathfrak{U})_{c}=\mathfrak{B}_{c}$, where $\nu=\nu_{\alpha}=\exp \left(-\pi / 4 \sqrt{-1} a d\left(X_{\alpha}+X_{-\alpha}\right)\right)$, and $X_{\alpha}$, $X_{-\alpha}$ are root vectors for $\alpha$ and $-\alpha$ in $\mathfrak{Y}$ satisfying $\left[X_{\alpha}, X_{-\alpha}\right]=$ $2 H_{\alpha} /\|\alpha\|^{2}$. In this case $[\mathfrak{U}] \neq[\mathfrak{B}]$, and $\nu \alpha$ is a singular imaginary root of $\mathfrak{B}$. Extending the order $<$ transitively we get an order on Car[פ] with unique maximal and minimal elements, the Cartan subalgebras with maximal compact and vector parts respectively.

For any tempered invariant eigendistribution $\pi$, let $C(\pi)$ be the subset of $\operatorname{Car}(G)$ consisting of conjugacy classes $[H]$ for which $\left.\pi^{\prime}\right|_{H} \neq 0$. Any Cartan subgroup $H$ such that $[H]$ is a maximal element of $C(\pi)$ is called a highest Cartan subgroup of $\pi$, and the function $k^{H}=\left.\varepsilon_{R}^{H} \Delta^{H} \pi^{\prime}\right|_{H}$ on $H$ is called one of the highest parts of $\pi$. $\pi$ is called extremal of height $[H]$ if $[H]$ is the unique maximal element of $C(\pi)$.

Let $\lambda$ be a homomorphism of $\mathscr{Z}$ into $C$. Let $H$ be a Cartan subgroup of $G$ with Lie algebra $\mathfrak{S}_{\text {, }}$ and complexification $\mathfrak{F}_{c}$. There is an isomorphism $\gamma: \mathscr{Z} \rightarrow I\left(\mathscr{F}_{c}\right)$, where $I\left(\mathscr{F}_{c}\right)$ denotes the invariants of the full Weyl group, $W\left(\mathfrak{Y}_{c}, \mathscr{F}_{c}\right)$, in $S\left(\mathscr{S}_{c}\right)$, the symmetric algebra of $\mathscr{S}_{c}\left[5\right.$, vol, I, p. 168]. Then $\lambda \cdot \gamma^{-1}=\lambda^{\mathfrak{s}}$ is a homomorphism of $I\left(\mathscr{S}_{c}\right)$ into $\boldsymbol{C}$ and is induced by a linear form $\mu$ on $\mathscr{S}_{c}$. We denote this relationship between $\lambda$ and $\mu$ by $\lambda^{\mathfrak{F}}=\lambda_{\mu}$. Clearly $\lambda_{\mu}=\lambda_{w^{\mu}}$ for all $w \in W\left(\mathfrak{Y}_{c}, \mathfrak{S}_{c}\right)$. 
For $H$ and $\lambda$ as above, let $B_{T}(H, \lambda)$ be the set of all analytic functions $\xi$ on $H$ such that:

(i) $D \xi=\lambda^{\mathfrak{f}}(D) \xi, D \in I\left(\mathfrak{S}_{c}\right)$;

(ii) $\xi(w h)=\varepsilon(w, h) \xi(h), w \in W(G, H)=N_{G}(H) / C_{G}(H), h \in H^{\prime}$, and $\varepsilon(w, h)= \pm 1$ is defined by $\varepsilon_{R}^{H}(w h) \Delta^{H}(w h)=\varepsilon(w, h) \varepsilon_{R}^{H}(h) \Delta^{H}(h)$;

(iii) $\sup _{h \in H}(1+\sigma(h))^{-s}|\xi(h)|<+\infty$ for some positive number $s$, where $\sigma$ is the function on $G$ defined in [5, vol. II, p. 66].

The following are proved by Hirai in [4].

Theorem 1. Let $\pi \in A_{T}(\lambda)$, and $H$ one of its highest Cartan subgroups. Then the highest part $k^{H}$ of $\pi$ on $H$ is a nonzero element of $B_{T}(H, \lambda)$. Conversely, for any nonzero $\xi$ in $B_{T}(H, \lambda)$, there exists at least one extremal element in $A_{T}(\lambda)$ with highest part $\xi$. The space $A_{T}(\lambda)$ is spanned by extremal elements.

Corollary 2. Suppose $B_{T}(H, \lambda) \neq\{0\}$. Every extremal element of $A_{T}(\lambda)$ with height $[H]$ is uniquely determined by its highest part if and only if $B_{T}\left(H^{\prime}, \lambda\right)=\{0\}$ for any $\left[H^{\prime}\right] \in \operatorname{Car}(G)$ such that $\left[H^{\prime}\right]<[H]$.

Lemma 3. In order that $B_{T}(H, \lambda) \neq\{0\}$ it is necessary that:

(i) there exist $\mu \in \sqrt{-1} \mathcal{S}^{*}$ such that $\lambda^{\mathfrak{5}}=\lambda_{\mu}$;

(ii) $\exp X \rightarrow \exp (\mu(X))$ is a well-defined unitary character of the connected component of the identity in $H$;

(iii) $\langle\mu, \beta\rangle \neq 0$ for any compact root $\beta$ of $\mathfrak{S}$.

Further, in order that $B_{T}(H, \lambda) \neq\{0\}$ it is sufficient that in addition to (i) and (ii), we have:

(iii) ${ }^{\prime} \mu$ is $I$-regular; that is, $\langle\mu, \beta\rangle \neq 0$ for any imaginary root $\beta$ of $\mathfrak{S}$.

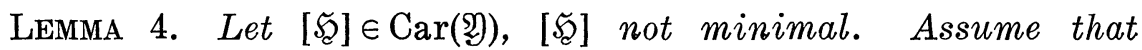
$\mu \in \sqrt{-1} \mathscr{S}^{*}, \quad \lambda_{\mu}=\lambda^{\mathfrak{s}}, \quad B_{T}(H, \lambda) \neq\{0\}$. Then in order that every extremal element of $A_{T}(\lambda)$ of height $[H]$ be uniquely determined by its highest part, it is necessary that $\mu$ be I-regular.

Finally, Hirai states the following lemma in the case that $\mathfrak{Y}$ is a simple real Lie algebra of classical type or of type $G_{2}$.

Lemma 5. Let $\mathfrak{Y}$ be a reductive real Lie algebra, and $\mathfrak{K}$ a Cartan subalgebra, not minimal with respect to the ordering on Car(פ). Let $\mu \in \sqrt{-1} \mathfrak{S}^{*}$ be I-regular, and let $\mathfrak{S E}^{\prime}$ be a Cartan subalgebra of $\mathfrak{Y}$ such that $\left[\mathfrak{S}^{\prime}\right]<[\mathfrak{S}]$. Let $\nu^{\prime}$ be any inner automorphism 
of $\mathfrak{Y}_{c}$ such that $\nu \mathfrak{S}_{c}=\mathfrak{S}_{c}^{\prime}$. Then $\nu \mu \in\left(\mathfrak{S}_{c}^{\prime}\right)^{*}$ is never pure imaginary on $\mathfrak{S}^{\prime}$.

The main result of this paper is that the above lemma is true as stated without restriction on the type of $\mathfrak{Y}$, and can be proved by a general argument. Using this lemma, together with Corollary 2 and Lemmas 3 and 4 , we obtain the following theorem.

THEOREM 6. Let $G$ be a connected, acceptable, real reductive Lie group with compact center. Let $H$ be a Cartan subgroup of $G$ with Lie algebra $\mathfrak{S}$, [S్E] not minimal in Car(Y). Assume that $B_{T}(H, \lambda) \neq\{0\}$, where $\lambda^{\mathfrak{s}}=\lambda_{\mu}, \mu \in \sqrt{-1} \mathcal{S}^{*}$. Then any extremal element $\pi$ in $A_{T}(\lambda)$ of height $[H]$ is uniquely determined by its highest part if and only if $\mu$ is I-regular.

3. Proof of Lemma 5. For any Lie algebra \&s and Cartan subalgebra $\mathfrak{F}_{c}$, let $\Phi=\Phi\left(\mathscr{S}_{c}, \mathscr{S}_{c}\right)$ denote the set of roots of the complexification, $\Phi_{R}, \Phi_{I}$, and $\Phi_{C P X}$ the subsets of $\Phi$ consisting of roots which are purely real, imaginary, or neither on $\mathfrak{S}$ respectively. For $\alpha \varepsilon \Phi, H_{\alpha}$ denotes the element of $\mathfrak{H}_{c}$ associated to $\alpha$ by the Killing form on $\mathfrak{S}_{c}, \mathscr{H}_{\alpha}$ the hyperplane in $\mathfrak{S}_{c}^{*}$ perpendicular to $\alpha$, and $s_{\alpha}$ the reflection through $\mathscr{H}_{\alpha}$. For $\gamma \in \Phi_{C P X}, \gamma^{\sigma}$ denotes the conjugate of $\gamma, \gamma^{\sigma}(H)=\overline{\gamma(\sigma(H))}, H \in \mathfrak{S}_{c}$, where $\sigma$ is the conjugation of $\mathbb{S}_{c}$ with respect to (\$). $\gamma_{I}$ and $\gamma_{R}$ denote the imaginary and real parts of $\gamma$ respectively.

Let $B=\Omega+\Re$ be a Cartan decomposition of $(S)$ with $\Re$ a maximal compact subalgebra for which $\mathfrak{S}=(\mathfrak{S} \cup \Re)+(\mathfrak{S} \cup \mathfrak{P})=\mathscr{S}_{k}+\mathfrak{S}_{p}$. Set $\sqrt{-1} \mathfrak{C}_{k}^{*}=\left\{\delta \in \sqrt{-1} \mathfrak{C}^{*}:\left.\delta\right|_{\mathfrak{q}_{p}}=0\right\}$, and $\mathfrak{S}_{p}^{*}=\left\{\delta \in \mathfrak{S}^{*}:\left.\delta\right|_{\mathfrak{F}_{k}}=0\right\}$.

Lemma 5 is proved by a series of reductions. First, by analyzing the inner automorphism $\nu: \mathfrak{S}_{c} \rightarrow \mathfrak{S C}_{c}^{\prime},\left[\mathfrak{S E}^{\prime}\right]<[\mathfrak{S}]$, we reduce the problem to a question just involving the root system $\Phi\left(\mathscr{S}_{c}, \mathscr{S}_{c}\right)$. We must prove that if $\mu \in \sqrt{-1} \mathscr{S}_{k}^{*}$ is $I$-regular, and if $w \mu \in \sqrt{-1} \mathfrak{C}_{k}^{*}$ for some $w \in W=W\left(\mathbb{S}_{c}, \mathfrak{S}_{c}\right)$, then $w \mu$ is also $I$-regular.

If $w \in W$ stabilizes $\Phi_{I}$, then clearly $w \mu$ is $I$-regular if and only if $\mu$ is. We examine the types of hyperplanes which can separate $\mu$ and $w \mu$. Since $\mu, w \mu \in \sqrt{-1} \mathcal{S}_{k}^{*}$, they cannot be separated by hyperplanes $\mathscr{C}_{\alpha}, \alpha \in \Phi_{R}$. We show that hyperplanes of the form $\mathscr{H}_{\beta}, \beta \in \Phi_{I}, \mathscr{H}_{r}, \gamma \in \Phi_{C P X},\left\langle\gamma, \gamma^{\sigma}\right\rangle \geqq 0$, and certain hyperplanes $\mathscr{H}_{\gamma}$, $\left\langle\gamma, \gamma^{\sigma}\right\rangle\left\langle 0\right.$, can be crossed by elements of $W$ which stabilize $\Phi_{I}$. Thus we may as well assume that $\mu$ and $w \mu$ are separated only by hyperplanes $\mathscr{H}_{\gamma},\left\langle\gamma, \gamma^{\sigma}\right\rangle\left\langle 0\right.$, such that $\gamma+\gamma^{\sigma}=\alpha \in \Phi_{R}$ is orthogonal to every other real root. 
This allows us to reduce the problem to a root system $\Phi^{\prime}$ for which $\Phi_{R}^{\prime}$ is of type $\left(A_{1}\right)^{r}$ for some integer $r$. We then show that the assumption that $w \mu$ is not $I$-regular leads to a contradiction.

Let $\mu \in \sqrt{-1} \mathfrak{S}^{*}$ be $I$-regular, and write $\mu=\mu_{k}+\sqrt{-1} \mu_{p}, \mu_{k} \epsilon$ $\sqrt{-1} \mathscr{S}_{k}^{*}, \mu_{p} \in \mathfrak{S}_{p}^{*}$. Then $\mu_{k}$ is also $I$-regular. For any $\left[\mathfrak{S}^{\prime}\right]<\left[\mathfrak{S}_{\mathcal{E}}\right]$ and inner automorphism $\nu$ such that $\nu \mathfrak{S}_{c}=\mathfrak{S}_{c}^{\prime}, \nu=\nu_{0} w$ for some $w \in W$ and some inner automorphism $\nu_{0}$ of $\mathscr{S}_{c}$ satisfying $\nu_{0} \mathfrak{K}_{c}=\mathfrak{S}_{c}^{\prime}, \nu_{0} \beta_{0}$ is a real root of $\mathfrak{S E}^{\prime}$ for some singular imaginary root $\beta_{0}$ of $\mathfrak{E}$, and $\nu_{0} \mathfrak{S}_{p} \subset \mathfrak{S}^{\prime}$.

Suppose for some $\mathfrak{S}^{\prime}$ and some inner automorphism $\nu=\nu_{0} w$ as above, $\nu \mu \in \sqrt{-1} \mathcal{S}^{\prime *}$. Then $\nu \mu_{k} \in \sqrt{-1} \mathfrak{S}^{*}$ also. Since $\mu_{k} \in \sqrt{-1} \mathfrak{Y}_{k}^{*}+$ $\mathfrak{S}_{p}^{*}$, the real subspace of $\mathfrak{S}_{c}^{*}$ spanned by $\Phi, w \mu_{k} \in \sqrt{-1} \mathfrak{C}_{k}^{*}+\mathfrak{S}_{p}^{*}$ also. But $\nu_{0} \mathfrak{S}_{p} \subset \mathfrak{S}^{\prime}$, so if $w \mu_{k}$ is nonzero on $\mathfrak{S}_{p}, \nu_{0} w \mu_{k}=\nu \mu_{k}$ takes nonzero real values on $\mathfrak{S}^{\prime}$. Thus $w \mu_{k} \in \sqrt{-1} \mathfrak{S}_{k}^{*}$.

Since $w \mu_{k}, \beta_{0} \in \sqrt{-1} \mathcal{F}_{k}^{*},\left\langle w \mu_{k}, \beta_{0}\right\rangle$ is real. But $\left\langle w \mu_{k}, \beta_{0}\right\rangle=$ $w \mu_{k}\left(H_{\beta_{0}}\right)=\nu \mu_{k}\left(H_{\nu_{0} \beta_{0}}\right)$ where $H_{\nu_{0} \beta_{0}} \in \mathfrak{S C}^{\prime}$ since $\nu_{0} \beta_{0}$ is a real root of $\mathfrak{S E}^{\prime}$. Thus $\left\langle w \mu_{k}, \beta_{0}\right\rangle=0$. Thus to prove that $\nu \mu$ is never pure imaginary on $\mathscr{S}^{\prime}$, it is sufficient to prove that $w \mu_{k}$ is $I$-regular for any $w \in W$ for which $w \mu_{k} \in V \overline{-1} \mathfrak{S}_{k}^{*}$, and we may as well assume that $\mu=\mu_{k} \in$ $\sqrt{-1} \mathfrak{S}_{k}^{*}$.

Assume that for $w \in W, w \mu \in \sqrt{-1} \mathfrak{K}_{k}^{*}$. We can pick $w^{\prime} \in W_{I}$, the subgroup of $W$ generated by $s_{\beta}, \beta \in \Phi_{I}$, such that $w^{\prime} \mu$ and $w \mu$ are separated by no hyperplanes $\mathscr{H}_{\beta}, \beta \in \Phi_{I}$. Further, $w^{\prime} \mu \in \sqrt{-1} \mathscr{F}_{k}^{*}$ and is $I$-regular since $W_{I}$ stabilizes $V \overline{-1} \mathcal{S}_{k}^{*}$ and $\Phi_{I}$.

For $\gamma \in \Phi_{C P X},\langle\gamma, \delta\rangle=-\left\langle\gamma^{\sigma}, \delta\right\rangle$ for any $\delta \in \sqrt{-1} \mathcal{S}_{k}^{*}$, so that $\mathscr{H}_{\gamma}$ separates $w^{\prime} \mu$ and $w \mu$ if and only if $\mathscr{H}_{\gamma^{\sigma}}$ does. If $\left\langle\gamma, \gamma^{\sigma}\right\rangle>0$, $\gamma-\gamma^{\sigma} \in \Phi_{I}$ and $\mathscr{L}_{\gamma}$ and $\mathscr{C}_{\gamma \sigma}$ separate $w^{\prime} \mu$ and $w \mu$ if and only if $\mathscr{H}_{\gamma-\gamma^{\sigma}}$ does. Thus $w^{\prime} \mu$ and $w \mu$ are not separated by hyperplanes $\mathscr{H}_{\gamma},\left\langle\gamma, \gamma^{\sigma}\right\rangle>0$.

Suppose they are separated by hyperplanes $\mathscr{H}_{\gamma},\left\langle\gamma, \gamma^{\sigma}\right\rangle=0$. For such $\gamma, s_{\gamma} s_{\gamma}{ }^{\sigma}$ stabilizes $\sqrt{-1} \mathcal{S}_{k}^{*}$ and $\Phi_{I}$. Therefore we can choose $\gamma_{1}, \cdots, \gamma_{k} \in \Phi_{C P X}$ such that $\left\langle\gamma_{i}, \gamma_{i}^{\sigma}\right\rangle=0, i=1, \cdots, k$, and $w \mu$ and $s_{\gamma_{1}} s_{\gamma_{1}}{ } \cdots s_{\gamma_{k}} s_{\gamma_{k}}{ }^{\sigma} w^{\prime} \mu=w^{\prime \prime} w^{\prime} \mu$ are separated only by hyperplanes $\mathscr{H}_{\gamma}$, $\left\langle\gamma, \gamma^{\sigma}\right\rangle<0$. Again, $w^{\prime \prime} w^{\prime} \mu \in \sqrt{-1} \mathcal{S}_{k}^{*}$ and is I-regular. Thus we may assume that $\mu$ and $w \mu$ are separated only by hyperplanes $\mathscr{H}_{r}$, $\left\langle\gamma, \gamma^{\sigma}\right\rangle<0$.

For such a root $\gamma, \gamma+\gamma^{\sigma}=\alpha \in \Phi_{R}$, so $\left.\gamma=\alpha / 2+\gamma_{I},\left\|\gamma_{I}\right\|^{2}\right\rangle$ $\|\alpha\|^{2} / 4$. Using the facts that $2\left\langle\gamma, \gamma^{\sigma}\right\rangle /\langle\gamma, \gamma\rangle$ and $2\langle\alpha, \gamma\rangle \mid\langle\gamma, \gamma\rangle$ are integers, we find that $\|\alpha\|^{2}=\|\gamma\|^{2}$. Suppose there is $\alpha_{1} \in \Phi_{R}$ such that $\left\langle\alpha, \alpha_{1}\right\rangle\left\langle 0, \alpha_{1} \neq-\alpha\right.$. Then $\left\langle\alpha_{1}, \gamma\right\rangle\left\langle 0\right.$, so $\gamma_{1}=\alpha_{1}+\gamma \in \Phi$. If $\|\alpha\|^{2} \neq\left\|\alpha_{1}\right\|^{2}, 2\left\langle\alpha_{1}, \gamma\right\rangle \mid\left\langle\alpha_{1}, \alpha_{1}\right\rangle$ or $2\left\langle\alpha_{1}, \gamma\right\rangle /\langle\gamma, \gamma\rangle$ is not an integer. Thus $\|\alpha\|^{2}=\left\|\alpha_{1}\right\|^{2}$ so that $\left\langle\gamma_{1}, \gamma_{1}^{\sigma}\right\rangle=0$. For any $\delta \in \sqrt{-1} \mathfrak{C}_{k}^{*},\left\langle\gamma_{1}, \delta\right\rangle=$ $\langle\gamma, \delta\rangle$, so that if $\mathscr{H}_{\gamma}$ separates $\mu$ and $w \mu$, so does $\mathscr{H}_{\gamma_{1}}$. But this contradicts the assumption that $\mu$ and $w \mu$ are separated by no 
complex hyperplanes $\mathscr{H}_{\gamma_{1}},\left\langle\gamma_{1}, \gamma_{1}^{\sigma}\right\rangle=0$. Thus if $\mathscr{H}_{r}$ separates $\mu$ and $w \mu, \gamma_{R}=\alpha / 2, \alpha$ is orthogonal to every other positive real root.

Pick $\gamma_{1}, \cdots, \gamma_{k}$, a subset of the set of all $\gamma$ such that $\mathscr{H}_{\gamma}$ separates $\mu$ and $w \mu$, for which $w \mu=s_{\gamma_{1}} \cdots s_{\gamma_{k}} \mu$. Let $\alpha_{1}, \cdots \alpha_{r}$ be the distinct real roots which appear as $\gamma_{i}+\gamma_{i}^{\sigma}, i=1, \cdots, k$. Then $\mathfrak{S}_{p}=\sum_{i=1}^{r} \boldsymbol{R} H_{\alpha_{i}}+\mathfrak{S}_{p}^{1}$ where $\mathfrak{S}_{p}^{1}=\left\{H \in \mathfrak{S}_{p}: \alpha_{i}(H)=0, i=1, \cdots r\right\}$.

Let $\mathfrak{M}$ be the centralizer of $\mathfrak{S}_{p}^{1}$ in $\mathscr{S}$. Then $\mathfrak{M}$ is a reductive Lie algebra with Cartan subalgebra $\mathfrak{K}_{\mathcal{E}}$, and $s_{\gamma_{1}} \cdots s_{\gamma_{k}}$ stabilizes $\Phi^{\prime}=$ $\Phi\left(\mathfrak{M}_{c}, \mathscr{S}_{c}\right)$. Further, $\Phi_{R}^{\prime}=\left\{ \pm \alpha_{1}, \cdots, \pm \alpha_{k}\right\}$ and $\Phi_{I}^{\prime}=\Phi_{I}$. Let 3 be the centralizer in $\mathfrak{M}$ of $H_{\sqrt{ }-1 \mu}$. Then $\Phi\left(\mathcal{Z}_{c}, \mathscr{S}_{c}\right)=\Phi^{\prime}(\mu)=\left\{\alpha \in \Phi^{\prime}\right.$ : $\langle\alpha, \mu\rangle=0\}$, and $s_{\gamma_{1}} \cdots s_{\gamma_{k}} \Phi^{\prime}(\mu)=\Phi^{\prime}(w \mu)=\left\{\alpha \in \Phi^{\prime}:\langle\alpha, w \mu\rangle=0\right\}$. If $w \mu$ is not $I$-regular, i.e., $\langle w \mu, \beta\rangle=0$ for some $\beta \in \Phi_{I}$, then $\Phi^{\prime}(w \mu)$ contains at least $r+1$ mutually orthogonal roots, namely $\alpha_{1} \cdots, \alpha_{r}$, $\beta$, and hence so does $\Phi^{\prime}(\mu)$.

A real root system is called $\sigma$-normal if $\alpha-\alpha^{\sigma} \notin \Phi$ for every $\alpha \in \Phi$ [3]. $\Phi^{\prime}(\mu)$ contains $\pm \alpha_{1}, \cdots \pm \alpha_{r}$, some complex roots, but no imaginary roots since $\mu$ is $I$-regular. Since $\alpha-\alpha^{\sigma}$ takes purely imaginary values on $\mathfrak{S}$ for any $\alpha \in \Phi^{\prime}(\mu), \alpha-\alpha^{\sigma} \notin \Phi^{\prime}(\mu)$, and so $\Phi^{\prime}(\mu)$ is a $\sigma$-normal real root system.

Write $\Phi^{\prime}(\mu)=\Phi^{1} \cup \cdots \cup \Phi^{s}$ where $\Phi^{i}$ is simple, $1 \leqq i \leqq s$ and $s \leqq r$. Then each $\Phi^{i}$ is a simple, $\sigma$-normal real root system with $\Phi_{R}^{i}$ of type $\left(A_{1}\right)^{r_{i}}$ and no imaginary roots. The possible simple $\sigma$ normal real root systems are classified, and we see by scanning a table such as [5, vol I, p. 30-32] that $\Phi^{i}$ can only be a real form of type $A_{2 r_{i}}$ or $A_{2 r_{i}-1}$. In either case, $\Phi^{i}$ contains exactly $r_{i}$ mutually orthogonal roots, and thus $\Phi^{\prime}(\mu)$ contains only $r$ mutually orthogonal roots. Therefore, $w \mu$ must be $I$-regular.

\section{REFERENCES}

1. Harish-Chandra, Invariant eigendistributions on semisimple Lie groups, Trans. Amer. Math. Soc., 119 (1965), 457-508.

2. Discrete series for semisimple Lie groups, I. Acta Math., 113 (1965), 241-318.

3. T. Hirai, Invariant eigendistributions of Laplace operators on real simple Lie groups, II. General theory for semisimple Lie groups, to appear.

4. Invariant eigendistributions of Laplace operators on real simple Lie groups, III. Methods of construction for semisimple Lie groups, to appear.

5. G. Warner, Harmonic Analysis on Semi-simple Lie Groups, 2 volumes, SpringerVerlag, New York, 1972.

Received April 27, 1976 and in revised form July 6, 1976.

UNIVERSITY OF CHICAGO 


\section{PACIFIC JOURNAL OF MATHEMATICS}

EDITORS

RICHARD ARENS (Managing Editor)

University of California

Los Angeles, California 90024

R. A. BEAUMONT

University of Washington

Seattle, Washington 98105

J. DugundjI

Department of Mathematics

University of Southern California

Los Angeles, California 90007

D. Gilbarg and J. Milgram

Stanford University

Stanford, California 94305

\section{ASSOCIATE EDITORS}

E. F. BeCKENBACH

B. H. NeUMaNN

F. WOLF

K. YoSHIDA

\section{SUPPORTING INSTITUTIONS}

UNIVERSITY OF BRITISH COLUMBIA

CALIFORNIA INSTITUTE OF TECHNOLOGY

UNIVERSITY OF CALIFORNIA

MONTANA STATE UNIVERSITY

UNIVERSITY OF NEVADA

NEW MEXICO STATE UNIVERSITY

OREGON STATE UNIVERSITY

UNIVERSITY OF OREGON

OSAKA UNIVERSITY

\author{
UNIVERSITY OF SOUTHERN CALIFORNIA \\ STANFORD UNIVERSITY \\ UNIVERSITY OF TOKYO \\ UNIVERSITY OF UTAH \\ WASHINGTON STATE UNIVERSITY \\ UNIVERSITY OF WASHINGTON \\ AMERICAN MATHEMATICAL SOCIETY \\ NAVAL WEAPONS CENTER
}




\section{Pacific Journal of Mathematics \\ Vol. 67, No. $1 \quad$ January, 1976}

Gregory Wayne Brumfiel and John W. Morgan, Homotopy theoretic

consequences of $N$. Levitt's obstruction theory to transversality for

spherical fibrations . ................................ 1

Jacob Burbea, Total positivity of certain reproducing kernels ........... 101

Wai-Mee Ching, The structure of standard $C^{*}$-algebras and their

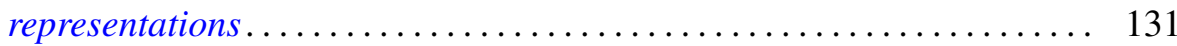

Satya Deo, The cohomological dimension of an $n$-manifold is $n+1 \ldots \ldots 155$

Masahiko Fujiwara and Masaki Sudo, Some forms of odd degree for which the Hasse principle fails ................................. 161

Mikihiro Hayashi, Smoothness of analytic functions at boundary points ... 171

Rebecca A. Herb, A uniqueness theorem for tempered invariant

eigendistributions ................................ 203

David Alan Legg, Orlicz space convergence of martingales of

Radon-Nikodým derivatives given a $\sigma$-lattice.................. 209

D. B. McAlister, v-prehomomorphisms on inverse semigroups......... 215

Bruno J. Mueller, Localization in fully bounded Noetherian rings . ........ 233

Donald J. Newman and A. R. Reddy, Rational approximation to $x^{n} \ldots \ldots .247$

Abraham Ziv, Inclusion relations between power methods of limitation.... 251 